\title{
Antibiotics prescreption pattern and resistance profile of common isolates at Soba university hospital in Khartoum, Sudan, a retrospective cross sectional study
}

Fatima Ahmed ( $\square$ fatimaAbdelraheem93@gmail.com )

faculty of medicine university of khartoum https://orcid.org/0000-0001-7749-0050

Abubaker Koko

Faculty of medicine, University of khartoum

Ola Mohamed

Soba University hospital

Reem Elhag

Soba university hospital

Sufian Khalifa

Faculty of medicine, Omdurman Islamic University

Mohamed Mohamed

Academy Charity hospital

Hind Abdelaziz

Academy Charity hospital

Almegadad Ahmed

Faculty of medicine, University of khartoum

Ihab Abdelrahman

Soba University Hospital / Faculty of Medicine University of Khartoum

Research

Keywords: Antibiotic, Hospital, Prescribing pattern, AMR, Sudan

Posted Date: April 22nd, 2020

DOl: https://doi.org/10.21203/rs.3.rs-22973/v1

License: (c) (1) This work is licensed under a Creative Commons Attribution 4.0 International License.

Read Full License 


\section{Antibiotics prescription pattern and resistance profile of common isolates at Soba university hospital in Khartoum, Sudan, a retrospective cross sectional study}

Fatima Abd Alraheem Ahmed ${ }^{1}$, Abubaker E.A Koko ${ }^{1}$, Ola Ahmed Abdulmjeed Mohammed ${ }^{2}$, Reem Yousif Elhag ${ }^{2}$, Sufian E.O. Khalifa ${ }^{3}$, Mohamed Elmojtaba Adil Mohamed ${ }^{4}$, Hind Abdelaziz Mohamed Abdelaziz ${ }^{4}$, Almegadad Sharafaldin Ahmed ${ }^{1}$, Ihab B. Abdelrahman ${ }^{2}$

1 Faculty of medicine, University of Kartoum (FatimaAbdelraheem93@gmail.com),(Bakri.imad.adlan@gmail.com),(almegdadsharaf@ gmail.com),

2 Soba university hospital (olamageed95@gmail.com)(reemyousif008@gmail.com), drtarawa1@hotmail.com,

3 faculty of medicine, Omdurman Islamic university (ports1997@ gmail.com)

4 Academy charity teaching hospital (hind991@gmail.com)(elmojtabaadel@gmail.com).

Corresponding author: Fatima Abd Alraheem Ahmed 


\begin{abstract}
Introduction: Antimicrobial resistance (AMR) is an increasingly global public health threat. An average of $45 \%$ of all patients are prescribed antibiotics. Nearly $50 \%$ of these prescribed antibiotics are unnecessary and they may promote the emergence of resistant strains. Antimicrobial stewardship programs have been introduced to tackle this problem. In this study we aim to determine the pattern of antibiotics prescription and resistance profile of common bacterial isolates at Soba university hospital, Sudan.

Methods: A retrospective cross sectional study that was conducted at the medicine and surgery wards in a tertiary care hospital. Records of all patients who were admitted during the study period were included. A pretested data collection form was used to collect information.
\end{abstract}

Results: Records of 269 inpatients were reviewed. The mean age of patients was $49.5 \pm$ 18.7 years. The most frequently reported diagnoses on admission included cerebrovascular accident and chronic liver disease, with $10.6 \%$ and $7.1 \%$, respectively. The mean duration of stay at the hospital was $6.6 \pm 6$ days. Almost $81.8 \%$ of admitted patients received antibiotics. The majority (38.7\% and $34.2 \%)$ were given one, and two antibiotics, respectively. Most of the antibiotics were administered intravenously (82.1\%). Third generation cephalosporin antibiotics (38\%) and metronidazole $(21.7 \%)$ were the most frequently prescribed. No documented indication for antibiotic prescription was found in $40.5 \%$ of cases. The main reason for stopping antibiotics was the patients' discharge $(63.6 \%)$. Of patients receiving antibiotics, only $5(1.9 \%)$ had undergone culture. Complete blood count (CBC) was requested in nearly all patients $(99.2 \%)$. A total of 100 cultures were performed during the study time. Most of them were urine cultures (64\%). Escherichia coli was isolated from 14 (29.2\%) cultures. Resistance percentage was highest for erythromycin, and oxacillin (100\% for both).

Conclusion: The study shows a trend of antibiotic misuse. Most patients received antibiotics without documented indication. Antibiotics sensitivity results showed high levels of resistance among isolated organisms. We recommend a wider study and formulation and implementation of clear guidelines to regulate antibiotic use.

Keywords: Antibiotic, Hospital, Prescribing pattern, AMR, Sudan 


\section{Introduction}

Antimicrobial resistance (AMR) is an increasingly global public health threat, that in the 21th century, the possibility of a post-antibiotic era in which common infectious diseases can kill is very tangible (1). Antimicrobial resistance has been linked directly via epidemiological studies to the consumption of antibiotics; misuse and overuse of these medicines is a substantial contributor to the antibiotic resistance (2-5). Over prescription of antibiotics results in applying a polarized, strong selective stress leading to the displacement of sensitive species and the rise of resistance ones $(6,7)$.

It is estimated that more than half of the world's medicines are sold, dispensed, or prescribed inappropriately (8). Additionally, reports indicated that two-thirds of antibiotic dispensing globally occur without prescription, and an average of $45 \%$ of all patients are prescribed antibiotics; this figure might rise up to over $70 \%$ of patients in some countries (9). Moreover, nearly $50 \%$ of these prescribed antibiotics are unnecessary and they may promote the emergence of resistant strains $(10,11)$. The magnitude of this issue increases in developing countries due to many factors including: underdeveloped health systems, unregulated over the counter sale of antibiotics, inappropriate use and poor prescription practices, and increased rates of antimicrobial resistance (12-14).

To tackle this issue, interventions have been introduced in order to optimize usage of antimicrobials, and encourage appropriate prescription practices, which are known collectively as antimicrobial stewardship programs (15). The goal of these interventions is a cost-effective usage of antimicrobials, while maximizing their therapeutic effects and decreasing their drug related toxicity and antimicrobial resistance (16).

Although the majority of antibiotic usage is within the community; increased antibiotic usage per patient, resistant pathogens, and infections due to resistant organisms are all concentrated more within hospital-settings $(17,18)$ Studies showed that antibiotic stewardship interventions in hospitals help to increase compliance with the local guidelines of prescriptions, and the clinical outcomes for patients (19-21). However, before organizing and implementing an antimicrobial stewardship program, information about prescription patterns and the situation of antimicrobial resistance need to be acquired (22).

In this study we aim to determine the pattern of antibiotics prescription and resistance profile of common bacterial isolates at Soba university hospital, Sudan.

\section{Methodology}




\section{Study setting and participants}

The is a retrospective cross sectional study that was conducted in Soba university teaching hospital, Khartoum, Sudan, which is a tertiary care hospital and the main teaching hospital for faculty of medicine, university of Khartoum. It consists of four major departments (internal medicine, surgery, pediatrics and obstetrics and gynecology). Total coverage of all patients who were admitted to the general medicine or surgery units at Soba university teaching hospital during August and September 2018 were included in the study.

\section{Data acquisition and analysis}

Data was collected from medical and laboratory records using well designed data collection sheets based on previous similar studies and it was pretested. Data sheets inquired about information regarding patients' sociodemographic characteristics, admission and hospital stay, antibiotics prescribed, and tests ordered. In addition, information regarding antibiotic sensitivity and culture results were also obtained retrospectively. Data was entered and analysed using statistical package for social sciences (SPSS) version 21.0. Descriptive statistics were conducted. Categorical data was described by frequencies and percentages, while continuous data was described using means and standard deviations (SD).

\section{Ethical consideration}

Ethical approval was obtained from the Soba Centre for Audit and Research, general director of soba university hospital, the statistic department and the head of laboratory unit at the hospital.

\section{Results}

The audit included the records of 269 patients, which was nearly halved between males (50.2\%), and females (49.8\%). The mean age of patients was 49.5 with a standard deviation (SD) of 18.7. Majority of patients were admitted to the internal medicine department $(65.1 \%)$, and nearly all of them $(96.7 \%)$ were admitted to the wards, with only $1.1 \%$, and $2.2 \%$ being admitted to the intensive care unit (ICU), and high dependency care unit (HDU), respectively.

The most frequently reported diagnoses on admission included cerebrovascular accident, chronic liver disease, malaria, and cholecystitis with $10.6 \%, 7.1 \%, 6 \%$, and $4.3 \%$, respectively. Duration of stay in the hospital ranged from 1 to 43 days, with a mean of 6.6 (SD =6).

Out of 269 patients admitted during the study period, 220 (81.8\%) received antibiotic prescription during their stay. Number of antibiotics prescribed for each patient ranged from 1 to 7 antibiotics, with the majority (38.7\%, and 34.2\%) given one, and two antibiotics, respectively. The total number of antibiotics prescribed amounted 350, most of which $(82.1 \%)$ were administered intravenously, with only $16.8 \%$, and $1.1 \%$ being oral, and intramuscular, respectively. Third generation cephalosporin antibiotics were the most frequently prescribed with 133 prescriptions (38\%), followed by metronidazole $76(21.7 \%)$, and second generation cephalosporin antibiotics 70 (20\%). The mean duration of antibiotic administration was 4.5 days $(\mathrm{SD}=3.5)$, ranging from 1 to 20 days. Table 1 illustrates details of antibiotics prescribed. 
Regarding indication of antibiotic prescription, over one third of patients who were prescribed antibiotics $(40.5 \%)$ did not have any documented indication, while prophylaxis, sepsis, urinary tract infection, and pneumonia were the most frequently documented indications, with $29.3 \%$, $10.3 \%, 6 \%$, and $5.6 \%$, respectively. Of the patients who were prescribed antibiotics, only 4 patients $(1.8 \%)$ developed complications related to the antibiotic's administration. The main reason for stopping antibiotics was the patients' discharge (63.6\%). Table 2 shows details of indications of antibiotic prescription.

Out of 224 patients who were prescribed antibiotics, only 5 (1.9\%) had undergone culture, and in all of them, culture sample was taken after starting the antibiotic. In addition, culture results were positive, and led to a change in the antibiotic prescribed in 4 patients. Klebsiella organism was isolated from 2 patients, while staph aureus, and methicillin resistant staphylococcus aureus (MRSA) were each isolated from 1 patient. Regarding other investigations, complete blood count (CBC) was requested in nearly all of patients $(99.2 \%)$, followed by renal function tests (RFT) (93.1\%), urinalysis (59\%), and liver function tests in $38.7 \%$ of patients. Table 3 details information of requested investigations.

We analyzed blood and urine culture results undertaken for patients in internal medicine, and surgery departments during the period of the study. A total of 100 cultures were performed, most of the them (86\%) from the internal medicine department, and majority of them $(64 \%)$ were urine cultures. About half of the cultures (49\%) were negative, and out of 51 positive cultures, 44 $(86.3 \%)$ isolated one organism, while $7(13.7 \%)$ isolated two organisms.

Total number of bacterial isolates was 48, while candida species was isolated in 9 cultures. Among bacterial isolates, Escherichia coli was isolated from 14 (29.2\%) cultures, Klebsiella pneumoniae from $10(20.8 \%)$, Enterococcus faecalis from 9 (18.8\%), and Pseudomonas aeruginosa from 7 (14.6\%). Two isolated organisms (Coccobacilli, and Klebsiella species) were resistant to all tested antibiotics. Resistance percentage was highest with erythromycin, and oxacillin (100\% for both), followed by cephalexin $(92.6 \%)$, cefuroxime $(85.7 \%)$, ceftriaxone $(82.6 \%)$, and tetracycline $(80 \%)$, while it was lowest with meropenem (12.5\%), vancomycin (14.3\%), amikacin (20.8\%), nitrofurantoin (24.1\%), and imipenem (25\%). Table 4 shows details of isolated strains and resistance.

\section{Discussion}

Antibiotic resistance is considered to be a warning global public health problem, as a result of inappropriate prescription, and misuse of antibiotics. In this study, our main objective was to determine the pattern of antibiotic prescription resistance profile of common bacterial isolates at Soba university hospital, Sudan.

In our study, $81.8 \%$ of patients received antibiotics during their admission, which is consider to be high in comparison with other studies in Nigeria, Canada, Ethiopia, Oman, and local studies in Elobeid, southern Sudan, and Khartoum, central Sudan which have reported prescription rates of $69.7 \%, 31 \%, 58.1 \%, 64 \%, 58.5 \%$, and $65 \%$, respectively. (22-27) 
Additionally, $82.1 \%$ of these prescribed antibiotics were administered intravenously, which is higher than what was reported in other different studies (28-30) .This high prevalence is alarming as parenteral antibiotics are costly, and the cost of medications is a major factor that may affect compliance to treatment in resource-limited countries $(22,26,28)$. Moreover, usage of parenteral routes requires more advanced level of training for health personnel, and it can increase the risk of transmission of infectious diseases (24).

Third generation cephalosporins and metronidazole were the most commonly prescribed antibiotics with an average of $38 \%$, and $21.7 \%$ respectively. This corresponds with findings of two similar studies in Nigeria, and another one in Elobied, southern Sudan in which third generation cephalosporins were the most commonly prescribed with an average of 34.5\% $(22,26,28)$ while a study from Oman reported that prescription of piperacillin/tazobactam, amoxicillin/clavulanic acid and clarithromycin predominated (25).

Over one third of patients in our study (40.5\%) lacked an indication for antibiotic prescription, which is considered another form of poor-prescribing practice (28). Also, there was poor documentation of the requested microbiological investigations as the patient's records showed that only $5(1.9 \%)$ patients had undergone culture, while the hospital laboratory reports in the same study period reported that there were 100 culture requests received from general medicine and surgery wards. In spite of this, the use of broad-spectrum antibiotics particularly cephalosporins predominates in this study, and this may be due to lack of diagnostic tools or poor utilization of available ones (22).

Of all patients to whom antibiotics were prescribed, cultures were conducted in only 5 (1.9\%). Compared to other studies form different countries, this percentage was considered the lowest; a study conducted in India reported that cultures were performed to only $11 \%$ of the patients who were given antibiotics; another study from India also stated that cultures were done to only $19 \%$ of patients $(31,32)$. Another study form Oman stated that only $25 \%$ of patients had undergone cultures before antibiotic administration (25). Moreover, a study from a tertiary referral center in Nigeria asserted that only $20 \%$ of their patients had undergone cultures before giving them antibiotics (28). In addition to low percentage of cultures, all the patients were already on antibiotics when their culture samples were taken, which might have altered the results of the cultures. Additionally, cultures were positive and led to a change in the prescribed antibiotic in 4 patients $(1.5 \%)$, compared to $12 \%$ in Oman (25). This reinforces that the ability of a hospital to perform cultures and sensitivity tests, is linked with its ability to implement rational antibiotic therapy (33).

The trends of antibiotic resistance were obtained from analysis of cultures results from the departments of internal medicine and surgery. A total of 100 cultures were perfumed, of them 48 were positive for bacterial isolates; the most commonly isolated organisms were Escherichia coli, Klebsiella pneumoniae, Enterococcus faecalis, and Pseudomonas aeruginosa. E. coli isolates showed high levels of resistance ranging from (79-93\%) to various antibiotics including third generations cephalosporins. This finding complements the results of a study conducted in a Sudanese hospital surgical wards in which the level of resistance was approximately 90\% (34). Similarly, another study performed in Khartoum state hospitals reported that $92 \%$ of E. coli 
isolates were MDR, and 53.3\% were resistant to more than 7 antimicrobial agents (35). Also, a study conducted to test the sensitivity of agents causing UTIs to the commonly used antibiotics in a Sudanese hospital revealed that E. coli isolates were $65 \%$ resistant to the 14 utilized antibiotics (36). Comparably, studies from other countries also reported high levels of resistance of E. coli isolates $(25,28,32)$. This high level of E. coli resistance in hospitals has many contributing factors including: lack of antimicrobial stewardships and strong infection control programs, and the easily available broad-spectrum antibiotics in the hospitals (37). Cultures were also positive for MRSA in this study, which is analogous to what has been discovered in a study performed in a Sudanese GIT surgery hospital, in which $71.4 \%$ of Staphylococcus aureus isolated were found to be MRSA (38).

\section{Conclusion}

This study revealed that most patients admitted to the medicine and surgery units during the study period have received antibiotics; majority of patients received more than one antibiotic, and most of them were administered parenterally. Additionally, third generation cephalosporin antibiotics were most commonly prescribed, and more than one-third of prescribed antibiotics were prescribed without documented indication. Furthermore, 100 cultures were performed during the study period; the most commonly isolated organisms were Escherichia coli and Klebsiella pneumoniae. Antibiotics sensitivity results showed high levels of resistance among isolated organisms, two of them (Coccobacilli, and Klebsiella species) were resistant to all tested antibiotics.

\section{Recommendation}

A larger multi-central study involving more departments and covering wider time period needs to be done. Antibiotic use audits should be a routine practice to ensure optimum antibiotic prescription practice. Clear guidelines should be formulated and implemented to prevent misuse of antibiotics and avoid resistance.

\section{List of abbreviations}

AMR: Antimicrobial resistance.

CBC: Complete blood count.

GIT: Gastrointestinal tract.

HDU: High dependency unit.

ICU: Intensive care unit.

MDR: Multidrug resistance.

MRSA: Methicillin resistance staphylococcus aureus.

RFT: Renal function test.

SD: Standard deviation. 
SPSS: Statistical package of social sciences.

UTIs: Urinary tract infections.

\section{Declarations}

\section{Ethics approval and consent to participate}

Ethical approval was obtained from the Soba Centre for Audit and research, general director of soba university hospital and the statistic department at the hospital.

\section{Consent for publication}

All authors are informed of and consent to submission of this manuscript.

\section{Availability of data and material}

The dataset collected and analyzed in the making of this manuscript is available from the corresponding author on demand.

\section{Competing interests}

All authors declare that they have no competing interests.

\section{Funding}

Not applicable.

\section{Authors' contributions}

Formulation of the idea and study design: IA, FA, OA, AK, MA; manuscript drafting: IA, OA, FA, AK, AA; data collection: OA, FA, AK, MA, SA, HM, RE; data analysis and interpretation: AK; manuscript writing: FA, AK, AA.

\section{Acknowledgments}

The authors would like to acknowledge Dr. Hana Albadawi, Lab Technician (laboratory unit, Soba university hospital), for her cooperation and invaluable advice.

\section{Authors' information}

Fatima A.O. Ahmed: Medical student, Khartoum university, FatimaAbdelraheem93@gmail.com. Abubaker E.A. Koko: Medical student, Khartoum university, Bakri.imad.adlan@gmail.com. Ola A. Abdulmjeed: Research fellow, Soba Center for Audit and Research (SCAR), Soba university hospital, olamageed95@gmail.com.

Sufian E.O. Khalifa: Medical student, Omdurman Islamic university, ports1997@gmail.com. Hind A.M. Abdelaziz: Medical officer, academy charity teaching hospital, hind991@gmail.com. Mohamed E.A. Mohamed: Teaching assistant, department of internal medicine, Omdurman Islamic university, Adeldrmohamed@gmail.com. 
Reem Yousif Elhag: Medical officer, Soba university hospital, (reemyousif008@ gmail.com).

Almegadad Sharafaldin Ahmed: Medical student, Khartoum university, (almegdadsharaf@gmail.com).

Ihab B. Abdelrahman: Associate professor of medicine, faculty of medicine university of Khartoum, (drtarawa1@ hotmail.com).

\section{References}

1. Organization WH. Antimicrobial resistance: global report on surveillance. World Health Organization; 2014.

2. The antibiotic alarm. Vol. 495, Nature. England; 2013. p. 141.

3. Ventola CL. The antibiotic resistance crisis: part 1: causes and threats. Pharm Ther. 2015;40(4):277.

4. Viswanathan VK. Off-label abuse of antibiotics by bacteria. Taylor \& Francis; 2014.

5. Lushniak BD. Antibiotic resistance: a public health crisis. Public Health Rep. 2014;129(4):314-6.

6. Michael CA, Dominey-Howes D, Labbate M. The antimicrobial resistance crisis: causes, consequences, and management. Front public Heal. 2014;2:145.

7. McGowan Jr JE. Antimicrobial resistance in hospital organisms and its relation to antibiotic use. Rev Infect Dis. 1983;5(6):1033-48.

8. Organization WH. Promoting rational use of medicines: core components. Geneva: World Health Organization; 2002.

9. World Health Organization. The world medicines situation. World Heal Organ. 2004;

10. Dellit TH, Owens RC, McGowan JE, Gerding DN, Weinstein RA, Burke JP, et al. Infectious Diseases Society of America and the Society for Healthcare Epidemiology of America guidelines for developing an institutional program to enhance antimicrobial stewardship. Clin Infect Dis. 2007;44(2):159-77.

11. Bronzwaer SLAM, Cars O, Buchholz U, Mölstad S, Goettsch W, Veldhuijzen IK, et al. The relationship between antimicrobial use and antimicrobial resistance in Europe. Emerg Infect Dis. 2002;8(3):278.

12. World trade organization, world health organization WIPO. Antimicrobial resistance: a global epidemic. 2017;

13. Stenehjem E, Hersh AL, Sheng X, Jones P, Buckel WR, Lloyd JF, et al. Antibiotic use in small community hospitals. Clin Infect Dis. 2016;63(10):1273-80.

14. Kimang'a AN. A situational analysis of antimicrobial drug resistance in Africa: are we losing the battle? Ethiop J Health Sci. 2012;22(2).

15. Organization WH. The evolving threat of antimicrobial resistance: options for action. Geneva: World Health Organization; 2012. 
16. Ganguly NK, Arora NK, Chandy SJ, Fairoze MN, Gill JP, Gupta U, et al. Global antibiotic resistance partnership (GARP): India Working Group. Rationalizing antibiotic use to limit antibiotic resistance in India. Indian J Med Res. 2011;134(3):281-94.

17. Golkar Z, Bagasra O, Pace DG. Bacteriophage therapy: a potential solution for the antibiotic resistance crisis. J Infect Dev Ctries. 2014;8(02):129-36.

18. French GL. The continuing crisis in antibiotic resistance. Int J Antimicrob Agents. 2010;36:S3-7.

19. Davey P, Brown E, Charani E, Fenelon L, Gould IM, Holmes A, et al. Interventions to improve antibiotic prescribing practices for hospital inpatients. Cochrane Database Syst Rev. 2013;(4).

20. Honda H, Ohmagari N, Tokuda Y, Mattar C, Warren DK. Antimicrobial stewardship in inpatient settings in the Asia Pacific Region: a systematic review and meta-analysis. Clin Infect Dis. 2017;64(suppl_2):S119-26.

21. Van Dijck C, Vlieghe E, Cox JA. Antibiotic stewardship interventions in hospitals in lowand middle-income countries: a systematic review. Bull World Health Organ. 2018;96(4):266.

22. Oduyebo OO, Olayinka AT, Iregbu KC, Versporten A, Goossens H, Nwajiobi-Princewill PI, et al. A point prevalence survey of antimicrobial prescribing in four Nigerian Tertiary Hospitals. Ann Trop Pathol. 2017;8(1):42.

23. Lee C, Walker SAN, Daneman N, Elligsen M, Palmay L, Coburn B, et al. Point prevalence survey of antimicrobial utilization in a Canadian tertiary-care teaching hospital. J Epidemiol Glob Health. 2015;5(2):143-50.

24. Desalegn AA. Assessment of drug use pattern using WHO prescribing indicators at Hawassa University teaching and referral hospital, south Ethiopia: A cross-sectional study. BMC Health Serv Res. 2013;13(1).

25. Al-Yamani A, Khamis F, Al-Zakwani I, Al-Noomani H, Al-Noomani J, Al-Abri S. Patterns of antimicrobial prescribing in a tertiary care hospital in Oman. Oman Med J. 2016;31(1):35-9.

26. Elfaki A. Assessment of Antibiotics prescription in Hospitalized Patients at Elobeid Hospital, Sudan. Sudan J Med Sci. 2009;4(3).

27. Awad AI, Himad HA. Drug-use practices in teaching hospitals of Khartoum State, Sudan. Eur J Clin Pharmacol. 2006;62(12):1087-93.

28. Iliyasu G, Dayyab FM, Bolaji TA, Habib ZG, Takwashe IM, Habib AG. Pattern of antibiotic prescription and resistance profile of common bacterial isolates in the internal medicine wards of a tertiary referral centre in Nigeria. J Glob Antimicrob Resist. 2015;3(2):91-4.

29. Tamuno I, Fadare JO. Drug prescription pattern in a Nigerian Tertiary hospital. Trop J Pharm Res. 2012;11(1):146-52. 
30. Wang Y-Y, Du P, Huang F, Li D-J, Gu J, Shen F-M, et al. Antimicrobial prescribing patterns in a large tertiary hospital in Shanghai, China. Int J Antimicrob Agents. 2016;48(6):666-73.

31. Nia SS, Hiremath SRR, Prasad S. Assessment of antimicrobial use pattern using world health organization prescribing indicators at a tertiary hospital: A prospective, observational study. J Appl Pharm Sci. 2018;8(6):132-8.

32. Rajalingam B, Susan Alex A, Godwin A, Cherian C, Cyriac C. Assessment of Rational Use of Antibiotics in a Private Tertiary Care Teaching Hospital. Indian J Pharm Pract. 2016;9(1):14-8.

33. Strengthening Pharmaceutical Systems.2012. How to Investigate Antimicrobial Use in Hospitals: SelectedIndicators.Published for the U.S. Agency for International Development by the Strengthening Pharmaceutical Systems Program. Arlington, VA: Management Sci.

34. Kheder SI. Cephalosporins usage and resistance trend in a Sudanese hospital surgical wards. J Pharm Biomed Sci. 2011;11(11).

35. Ibrahim ME, Bilal NE, Hamid ME. Increased multi-drug resistant Escherichia coli from hospitals in Khartoum state, Sudan. Afr Health Sci. 2012;12(3):368-75.

36. Saeed A, Hamid SA, Bayoumi M, Shanan S, Alouffi S, Alharbi SA, et al. Elevated antibiotic resistance of sudanese urinary tract infection bacteria. EXCLI J. 2017;16(2007):1073-80.

37. Aly M, Balkhy HH. The prevalence of antimicrobial resistance in clinical isolates from Gulf Corporation Council countries. Antimicrob Resist Infect Control. 2012;1(1):26.

38. Kheder SI, Ali NA, Fathelrahman AI. Prevalence and Antimicrobial Susceptibility Pattern of Methicillin Resistance Staphylococcus in a Sudanese Surgical Ward. Pharmacol \&amp; Pharm. 2012;03(01):103-8. 


\section{Supplementary Files}

This is a list of supplementary files associated with this preprint. Click to download.

- Tables.pdf 